\title{
Inversión estatal en investigación y desarrollo forestal frente a la COP-25: ¿Libres de elegir entre bosques nativos y plantaciones exóticas?
}

\author{
Public investment in research and development towards COP-25: Free to choose between native \\ forests and exotic plantations?
}

\author{
Historial del artículo \\ Recibido: \\ 25 de septiembre de 2019. \\ Aceptado:
}

30 de octubre de 2019

\section{Daniela Manuschevich $^{\mathrm{a}}$}

a Universidad Academia de Humanismo Cristiano, Santiago, Chile. Correo electrónico: dmanuschevich@academia.cl

\section{Palabras clave}

Bosques nativos, plantaciones exóticas, política forestal, sumideros de carbono

\section{Keywords}

Carbon sink, forest policy, native forests, tree farms

\begin{abstract}
Resumen
En el contexto de la conferencia de las Partes sobre Cambio Climático (COP25) se plantea que el sector forestal sería uno de los grandes sumideros de carbono que le permitirían a Chile disminuir la intensidad de sus emisiones de carbono. El gobierno plantea que las opciones de mitigación que ofrece el manejo de bosque nativo, junto con una renovada expansión de las plantaciones forestales contribuirían a esta meta. Ante esta propuesta cabe preguntarse cuanto ha invertido el Estado en desarrollar investigación, tecnología y desarrollo productivo de estas dos opciones. La hipótesis es que se ha invertido más en especies exóticas que en bosque nativo. Se elaboró y analizó una base de datos de proyectos financiados total o parcialmente por el Estado. Se analizaron 1.139 proyectos entre los años 1981 y 2018, los que se compararon en términos de los montos asignados, dinámicas temporales y espaciales. Se asignó en total 1,5 veces más fondos a proyectos de investigación y desarrollo en plantaciones forestales que a proyectos similares basados en especies nativas, siendo un proyecto de especies exóticas 1,6 más caro que su contraparte. Desde 2010, ha habido una tendencia al alza en proyectos sobre especies nativas, sin embargo, esto se sostiene únicamente en el Fondo de Investigación para el Bosque Nativo. Se discuten las implicancias de estas desigualdades de conocimiento y desarrollo. Se concluye que las diferencias en inversión y desarrollo en el sector forestal han ayudado a construir sector basado en plantaciones forestales muy rentables, en desmedro del desarrollo de los bosques nativos y sus territorios.
\end{abstract}




\section{Introducción}

En el contexto de la Conferencia de las Partes sobre Cambio Climático (COP25), se plantea que el sector forestal sería uno de los grandes sumideros de carbono. Chile debe presentar sus Contribuciones Nacionalmente Determinadas que le que le permitirían disminuir su huella de carbono por unidad de crecimiento y así cumplir con las metas de reducción de emisiones (Gobierno de Chile, 2015). Para alcanzar esta meta, el Ministerio de Agricultura impulsa un nuevo subsidio a la forestación por un monto anual de 37,5 millones de dólares. Este nuevo subsidio fomentaría las plantaciones tanto nativas como exóticas, sin diferencias normativas, pues se argumenta que los propietarios deben ser libres de decidir qué puede plantar (Guerra Martínez, 2019). Es sabido que las plantaciones forestales en Chile son muy rentables (Cubbage et al., 2014 2007), por lo que ante esta nueva propuesta de subsidio, resulta relevante preguntarse cuánto ha invertido el Estado en investigación y desarrollo en ambos sectores.

Para efectos de este artículo se entenderá como sector forestal toda la actividad económica que dependa de los bienes y servicios que producen las plantaciones y los bosques. Esto incluye producción de fibra para papel y madera, muebles, productos forestales no madereros, tales como aceites, frutos, hongos y esencias (FAO, 2004). Se entenderá como investigación y desarrollo como las actividades cuyo propósito en mejorar el estado y uso de los bosques y plantaciones (USFS, 2019).

Estados Unidos, Canadá, Alemania, Finlandia y Suecia son los países cuyo sector forestal más aporta al intercambio mundial, es decir, mayor valor de exportaciones (FAO, 2019). En general, en ninguno de estos cuatro países la exportación de celulosa supera el 28\% (Canadá), seguido por Finlandia y Suecia, con un 16\% y 13\%, respectivamente. En contraste, en Chile la celulosa constituye el $65 \%$ de las exportaciones en valor exportado en el año 2017 (FAO, 2019). El índice de complejidad económica, publicado regularmente por el Observatorio de Complejidad Económica, es un índice que indica la complejidad de la producción y se relaciona con el crecimiento a futuro y con la desigualdad de los países (Hartmann, Guevara, Jara-Figueroa, Aristarán \& Hidalgo, 2017; Hidalgo \& Hausmann, 2009). En este ránking la pulpa sulfatada de celulosa está ubicada en el sitio 598 de 1.232 productos que se estudian, es decir, no es un producto de alta complejidad (clasificación armonizada de cuatro dígitos HS 92). El exportador más grande de pulpa sulfatada de celulosa es Brasil, mientras Chile ocupa el tercer lugar de exportación mundial de este producto. Es decir, Chile exporta grandes cantidades de un producto de complejidad media a baja. En contraste, de todos los productos que se podrían obtener del bosque, los productos farmacéuticos derivados de flora, tales como perfumes, productos cosméticos y fungicidas derivados, estos son los más complejos. A nivel mundial, esta producción es liderada por México (Simoes \& Hidalgo, 2011).

En cuanto a institucionalidad forestal para investigación y desarrollo, los cuatro países mencionados cuentan con un sistema estatal de investigación y desarrollo. En Estados Unidos el Servicio Forestal (USFS) se creó en 1901, pero el área de investigación se creó en 1876 dentro del Departamento de Agricultura. Actualmente, el USFS cuenta con ochenta y un bosques experimentales en todo el país, contando con 500 investigadores permanentes, 446 publicaciones con bases de datos abiertas, que varían desde cobertura vegetacional hasta información socioeconómica de los propietarios de bosques (USFS, 2019). En Canadá, el Servicio Forestal federal existe desde 1899 y cuenta con cinco estaciones de investigación, dos bosques dedicados a la investigación y siete centros de investigación temática, tales como cambio climático, mejoramiento del papel, bioenergía, que en algunos casos contiene otros subcentros regionales. También cuenta con base de datos de publicaciones científicas y bases de datos abiertas, ya que la investigación es de carácter público (Goverment of Canada, 2019). Alemania es conocida por su larga tradición de uso de los bosques y por generar las primeras escuelas forestales ligadas a una visión estratégica de los bosques como proveedor de madera para actividades económicas internas y como recurso estratégico durante las guerras (Fernow, 1911). Alemania tiene un sistema federado que tiene agencias de investigación de acuerdo con su administración territorial que redunda en al menos diez centros de investigación forestal, más una autoridad nacional. Además, el Ministerio de Alimentos y Agricultura cuenta con el Instituto Federal Yohann Von Thünen de investigación en áreas rurales, forestal y pesca dedicado específicamente a hacer monitoreo de largo plazo, apoyar la toma decisiones, políticas y regulaciones (BMEL, 2019; Thünen Institute, 2019).

Todos los países mencionados basan sus industrias principalmente en especies nativas. En general, todas las agencias de gobierno cuentan con un área dedicada a monitoreo y sistema de información geográfica, y un área de estudios sobre bosques y cambio climático de carácter y financiamiento permanente. Además, estos países cuentan con un área de investigación en cambio climático, bioeconomía o economías de transición. Es sabido ya que los retornos sociales de la investigación realizadas 
por entes públicos exceden los retornos privados, que en caso de la agricultura pueden llegar hasta el 35\% (Arrow, 1962; Fuglie et al., 1996), por lo que el financiamiento público es fundamental.

En contraste, en Chile la institucionalidad a cargo de la información catastral, investigación y desarrollo son la Corporación Forestal (CONAF), el Instituto Forestal (INFOR) y el Centro de Información de Recursos Naturales (CIREN). Todas estas son de derecho privado. Si bien reciben presupuesto público, dependen en distinto grado de ayudas privadas y venta de servicios. En el caso de CONAF, que está encargada de monitorear las emisiones por cambio de uso de suelo, este monitoreo es licitado, año a año, lo que dificulta la comparación del cambio en el uso del suelo y por lo tanto de las emisiones relacionadas con este fenómeno (Miranda et al., 2018). Así mismo, se licita la ejecución de una multiplicidad de proyectos relacionados con cambio climático, lo que dificulta el aprendizaje y memoria institucional. CIREN (2012), que posee bases de datos completas de suelos y predios, hasta hace poco vendía dicha información, lo que impide el acceso a quien no tenga el capital. La competencia por fondos por otra parte hace que las agencias encargadas de monitoreo, investigación y desarrollo compitan por fondos de investigación pública, lo cual dificulta la continuidad de los proyectos de desarrollo, que en general requieren largos horizontes y por lo tanto requieren del apoyo sustantivo del Estado (Carmona et al., 2010; Kile, Nambiar \& Brown, 2014).

La gobernanza descrita anteriormente, combinado con un sector forestal basado en plantaciones forestales muy rentable, redunda en profundas transformaciones socioambientales que han ocurrido en Chile sur-central a pesar de los intentos regulatorios (Miranda, Altamirano, Cayuela, Lara \& González, 2017). Estas plantaciones forestales se expandieron sobre vegetación nativa y, más recientemente, sobre vegetación arbustiva y agrícola (Echeverria et al., 2006; Heilmayr, Echeverría, Fuentes \& Lambin, 2016; Miranda et al., 2017). La expansión de las plantaciones forestales ha tenido impactos negativos de desarrollo territorial y están estadísticamente relacionadas con mayor pobreza y emigración, así como a incendios forestales, a la vez que con el despojo material y simbólico de comunidades indígenas (Andersson, Lawrence, Zavaleta \& Guariguata, 2016; Cárdenas \& Antileo, 2006; Gerber, 2011; McWethy et al., 2018).

En el contexto chileno, donde ya se han plantado casi tres millones de hectáreas con plantaciones industriales por cuarenta años, la forestación no se trata simplemente de una política sencilla para mitigar el cambio climático mediante el secuestro de carbono. La forestación plantea el desafío de hacerlo al considerar que estos bosques deben generar un sustento para que las personas puedan vivir allí en un contexto de cambio climático, es decir, menor precipitación y mayores temperaturas. Este es el desafío de política pública y el verdadero reto para el desarrollo tecnológico sostenible. De no acompañar alternativas de sustento para las familias campesinas se exacerba el despoblamiento del campo y la migración a la ciudad con los problemas para la estabilidad social que esto conlleva, lo que es conocido en la literatura como "la cuestión agraria" (Bernstein, 2002; Clapp, 1998). De acuerdo a Franco \& Borras (2019), el actual incremento rápido de demandas por tierra para mitigación y adaptación climática, altera el acceso y control a la tierra exacerbando los problemas que plantea la cuestión agraria (Borras \& Franco, 2018; Franco \& Borras, 2019). En resumen, es importante diferenciar las políticas de mitigación para campesinos y pequeños propietarios, de las grandes extensiones de plantaciones industriales.

Desde la perspectiva de la economía neoclásica, el uso del suelo es el resultado de condiciones naturales y de la libre decisión de los propietarios que toman en consideración las condiciones del suelo, el estado de los mercados y los costos, entre otros factores, para luego decidir cómo utilizarán su propiedad (ver, por ejemplo, Mather \& Needle, 1998). Sin embargo, Mansfield, Munroe \& McSweeney (2010) argumenta que el cambio en el uso del suelo es el resultado de relaciones sociales y económicas entre regiones del mundo, las que están mediadas por relaciones económicas y agentes que facilitan o dificultan estas interacciones. Munroe (2014) plantea que el entendimiento del cambio en el uso del suelo, desde una perspectiva geográfica, debe apuntar a entender este fenómeno como socialmente embebido, para prestar atención a los procesos históricos y de cómo influencian lo que hoy se puede conceptualizar como el mercado de la tierra y sus opciones de uso. De esta forma, se puede llegar a comprender cómo es que fenómenos espaciales como el cambio en el uso del suelo han llegado a ocurrir.

En este mismo sentido, Peck (2001) argumenta que, contrario a lo que se tiende a ilustrar, los Estados neoliberales no son menos intervencionistas que otros tipos de Estado. Por el contrario, los Estados neoliberales organizan y racionalizan las intervenciones de manera tal de mantener el control de sectores clave, para asegurar, por ejemplo, la mantención de las condiciones de suministro de material para los mercados internacionales. En el discurso, se plantea que son los mercados son los que deciden qué es lo que se 
Tabla 1

Fuentes de información desde donde se recopiló la información del presente trabajo.

\begin{tabular}{llll}
\hline Agencia & Fuente & Palabra de búsqueda & Período de datos \\
\hline $\begin{array}{l}\text { Observatorio para la Innovación } \\
\text { Agraria, Agroalimentaria y Forestal }\end{array}$ & Transparencia pasiva en base OPIA & Bosque nativo o forestal & 2008-2019 \\
\hline CORFO & Transparencia activa & Forestal & $\begin{array}{l}\text { Forestal o Forestry en categorización OCDE/ 2018 } \\
\text { disciplina/ nombre del proyecto/ }\end{array}$ \\
\hline CONICYT & Transparencia activa & Sección de transparencia página web 2018 & $2016-2018$ \\
\hline INFOR & Transparencia pasiva & &
\end{tabular}

Fuente: Elaboración propia.

produce, pero es el Estado quien ejerce su influencia en la presencia de sectores clave, como por ejemplo el caso del área de investigación, conocimiento y tecnología, ya que tiene un horizonte de largo plazo, con niveles de alta inversión y riesgo asociado del cual difícilmente algún ente privado se haría cargo (Kile et al., 2014). Es así como el desarrollo de la industria forestal de Australia se logró, por ejemplo, solamente gracias al fuerte y constante apoyo del Estado en la entrega de fondos y la coordinación a la industria, universidades y entes gubernamentales (Kile et al., 2014). Se plantea entonces examinar la inversión que ha hecho el Estado en dos sectores que hoy resultan críticos para la mitigación de las emisiones de carbono: bosques nativos y plantaciones forestales.

En este trabajo se parte de la premisa que las opciones a las que pueda acceder un pequeño o mediano propietario están fuertemente relacionadas con la inversión estatal en investigación y desarrollo. Por ejemplo, si existe poco conocimiento masificado sobre el cultivo de especies como el coihue (Nothofagus dombeyi), es poco probable que esta especie sea seleccionada por un pequeño propietario, a pesar de que puede ser la mejor opción en el largo plazo. En contraste, es mucho más probable que se seleccione una especie abundante en los viveros del país, un esquema de manejo y crecimiento muy conocido y masificado, con un posible comprador claro, como hoy ocurre con la venta de madera, fibra, y leña de eucaliptus o pino.

En vista de todos estos antecedentes, el objetivo de este trabajo es saber cuánto ha invertido el Estado en investigación y desarrollo en especies nativas, principalmente forestales, y en especies exóticas, principalmente especies de rápido crecimiento. El foco de este trabajo no está en el propósito de la actividad de investigación o desarrollo, sino que en el origen biogeográfico de la especie. Es decir, se consideran tanto actividades de conservación como de producción. De esta forma se busca comenzar a comprender qué opciones efectivamente puede tener un pequeño o mediano propietario al plantar. La hipótesis de este trabajo es que se ha invertido más en investigación y desarrollo de especies exóticas que nativas.

\section{Metodología}

Se realizó una búsqueda en la base de datos de proyectos del Observatorio para la Innovación Agraria, Agroalimentaria y Forestal (OPIA) por sus siglas ( (FIA, 2019). La búsqueda se realizó con la palabra "forestal" y otra búsqueda con "bosque nativo". Esta base de datos contiene información sobre el proyecto, año, región, especie forestal, fuente de financiamiento, región de ejecución, código del fondo de aporte, aporte de contrapartes, monto total, rubro y tema. Para asegurar que los datos fueran más completos y precisos, se revisaron además las fuentes de información que se indican en la Tabla 1. Como este estudio se enfocó en la inversión estatal de fondos se eliminaron los proyectos de financiamiento privado o internacional, tales como empresas o agencias internacionales.

La base de OPIA contiene todos los proyectos del Fondo de Investigación del Bosque Nativo administrado por CONAF, gran parte de los proyectos de CORFO y de CONICYT, por lo que la búsqueda se complementó con las siguientes búsquedas:

CORFO - Corporación de Fomento de la producción. Se solicitó por transparencia activa la lista de todos los proyectos que contuvieran la palabra "forestal". Se clasificaron los proyectos de acuerdo con el esquema de codificación indicado en el apéndice. Se categorizaron los proyectos en base al nombre del proyecto en caso de que esta información no hubiese sido claramente establecida en la variable de rubro o producto del beneficiario del proyecto. En caso de no existir información clara con ninguno de los dos criterios anteriormente mencionados se excluyó el proyecto. Se asumió que cuando el título de proyecto no fue claro, el rubro de la empresa beneficiada nos indica la temática del proyecto. 


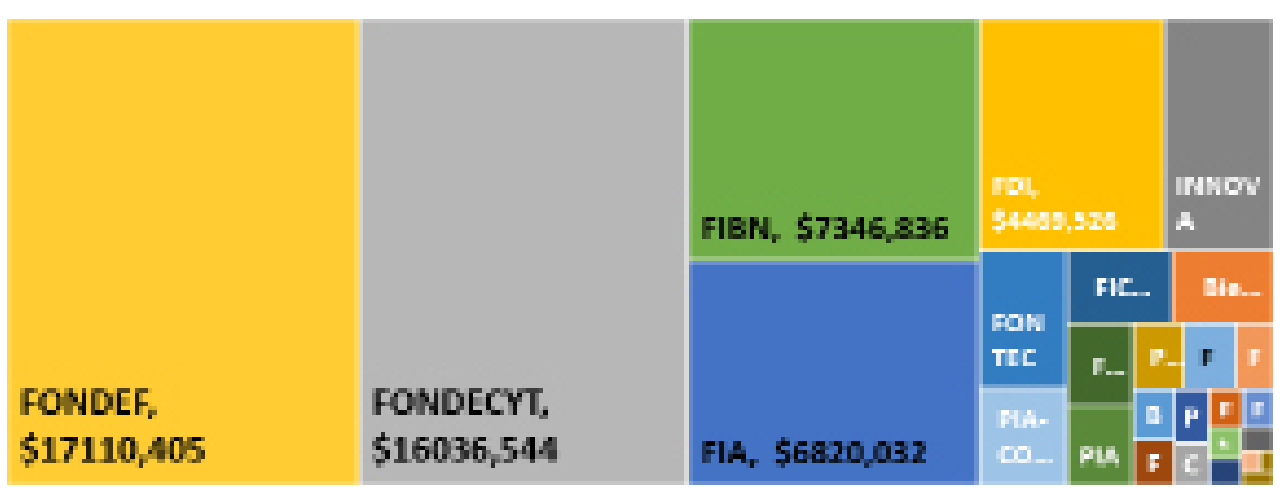

Figura 1. Fuentes de financiamiento en millones de pesos ajustados por inflación para proyectos centrados en especies nativas (1981-2018). Fuente: Elaboración propia.

CONICYT- Comisión Nacional de Investigación Cientifica y Tecnológica. Se solicitó por transparencia activa todos los proyectos que contuvieran la palabra "forestal". La búsqueda se centró en la disciplina OCDE de ciencias agrícolas y se buscaron aquellos proyectos que tuvieran la palabra "forestal" en ellos. Se codificó con el mismo esquema de la sección anterior, es decir, las entradas se codificaron nuevamente para excluir los que tuvieran la palabra "forestal", pero que no fueran de interés.

INFOR-Instituto forestal. Se consideraron todos los proyectos publicados por transparencia activa en el sitio web de INFOR. Solo estaban disponibles los proyectos desde el 2016 a 2019. Se excluyeron los proyectos de CORFO, CONICYT, FIA y CONAF, por encontrarse en las bases de datos anteriormente mencionadas.

Codificación. Se construyó una base de datos con 1.139 proyectos asignados hasta 2018 . Todos los montos se manejaron en base a millones ajustados por la inflación anual promedio desde 1981 obtenida desde la base de datos de inflación del Banco Mundial (World Bank, 2018), se usó como año base el 2018. Se clasificaron los proyectos de acuerdo con el esquema de codificación indicado en el apéndice, es decir: especies nativas, exóticas y proyectos que trataran ambos grupos. Se calculó el costo promedio por proyecto invertido en los sectores de bosque nativo, plantaciones forestales y en proyectos que trataran ambos temas. Luego, se clasificaron los proyectos según períodos presidenciales, que se consideró hasta el año en que asume una mandataria o mandatario. Por ejemplo, el período de Ricardo Lagos comprende los años completos de 2001 hasta 2006, pues se asume que la reorientación de políticas no es inmediata, sino que demora algunos meses.
En cuanto a la región de ejecución del proyecto, solo fue posible establecerla en un $67 \%$ de los casos, por lo que los resultados de esta sección se centran en esa parte de la muestra. No se consideró la región del Ñuble como una región aparte porque esta se creó el 2018, que es el último año de la base de datos con la que se trabajó. Si se consideró la región de los Ríos, pero puede ser que la cantidad de proyectos se encuentre subestimada, por la fecha en la que se creó (2007).

\section{Resultados}

\section{Inversión estatal por sector}

Entre 1981 y 2018 , se asignaron US\$ $246,660,140^{1}$ en montos totales para investigación y desarrollo en este sector ( $\$ 176.362$ millones de pesos $\left.{ }^{2}\right)$. De este total, el $53 \%$ de los fondos se asignaron a plantaciones, un 35\% a bosques nativos y un $12 \%$ a proyectos que trataran ambas temáticas. El Estado chileno invirtió 1,5 veces más en proyectos relacionados con plantaciones forestales que en proyectos centrados en bosques nativos. El monto promedio de los proyectos destinados al bosque nativo fue cercano a los $\$ 116$ millones, mientras que un proyecto de plantaciones costó $\$ 192$ y en $\$ 175$ en proyectos que involucren a ambos.

En cuanto al aporte de distintos fondos para cada uno de los sectores, en el sector de especies y bosques nativos, el fondo más importante fue FONDEF, que entregó un $28 \%$ del financiamiento, seguido por FONDECYT, con el 26\%. El Fondo de Investigación del Bosque Nativo (FIBN) de CONAF es el tercer fondo con un $15 \%$ de los fondos asignados (Fig. 1). Le siguen el Fondo de

\footnotetext{
${ }^{1}$ Se usó una conversión de un dólar a $\$ 715$ pesos chilenos.

${ }^{2}$ Este monto es ligeramente inferior a la concesión de la ruta Nahuelbuta, la cual uniría Los Ángeles con Angol en $55 \mathrm{~km}$ y tiene un costo total de 6.125 UF.
} 


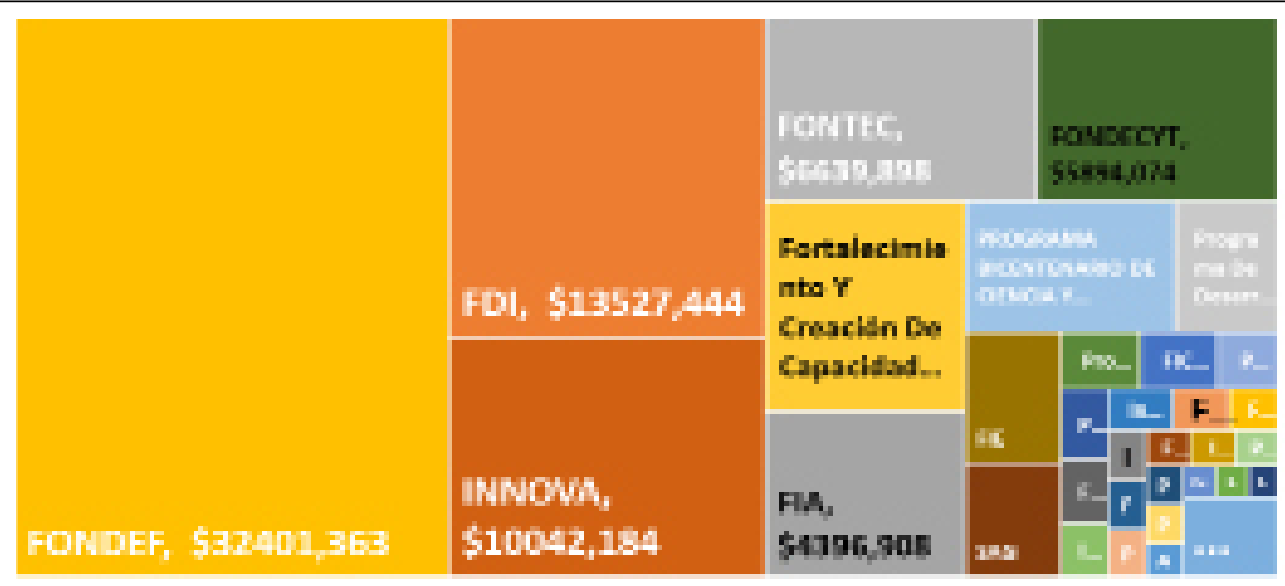

Figura 2. Fuentes de financiamiento en millones de pesos ajustados por inflación para proyectos centrados en especies exóticas (1981-2018). Fuente: Elaboración propia.

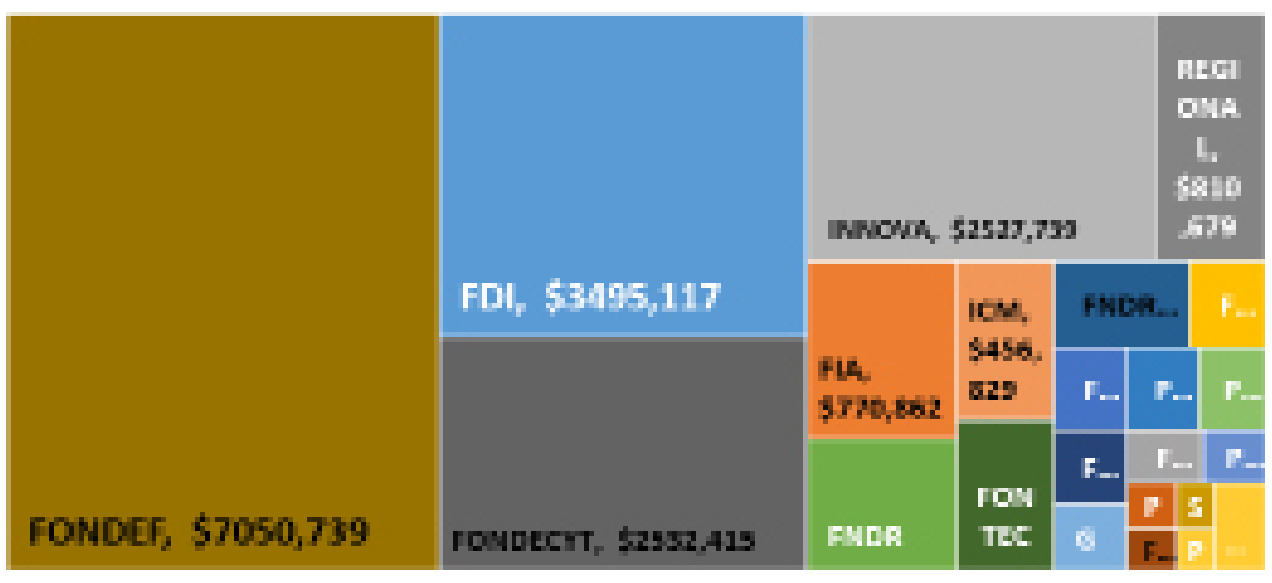

Figura 3. Fuentes de financiamiento de proyectos en millones de pesos ajustados por inflación relacionados con especies nativas y exóticas (1981-2018). Fuente: Elaboración propia.

Innovación Agraria (FIA) y el Fondo de Desarrollo e Innovación (FDI) de CORFO.

En el sector de especies exóticas, el fondo más importante fue FONDEF, con un $34 \%$ de los fondos asignados, seguido por FDI e INNOVA, ambos programas de CORFO, con un 14 y $11 \%$ de los fondos, respectivamente (Fig.2). El programa FONTEC, que fue el precursor del programa INNOVA, es el cuarto fondo más importante. Si sumamos los montos de FONTEC más INNOVA, CORFO aportó un $18 \%$ de los fondos mediante estos dos instrumentos. Por último, los proyectos financiados por FONDECYT ocupan un lugar más secundario, con el $6 \%$ de los fondos asignados (Fig.2).

En cuanto a los proyectos centrados en bosque y en plantaciones exóticas (Fig. 3), los fondos más importantes provienen de FONDEF, Fondo de Desarrollo e Innovación (FDI), FONDECYT e INNOVA, con un 34,17 y $12 \%$ de los fondos, respectivamente. En este sector, hay fuentes de financiamiento que no aparecen en los otros sectores como los fondos regionales, que juntos dan cuenta de un $12 \%$ del financiamiento. También son más relevantes las Iniciativas Científicas Milenio (ICM), que en este caso dan cuenta de un $3 \%$ de los fondos asignados.

\section{Desarrollo temporal de la inversión}

Los fondos asignados a investigación y desarrollo en todos los sectores han aumentado sostenidamente desde 1981 a la fecha. De acuerdo con los datos recopilados, en 1981 se invirtieron solo US $\$ 5,711,888$ (\$4.084 millones de pesos chilenos), mientras que en 2018 se invirtieron US\$13,262,937 (\$9.483 millones 


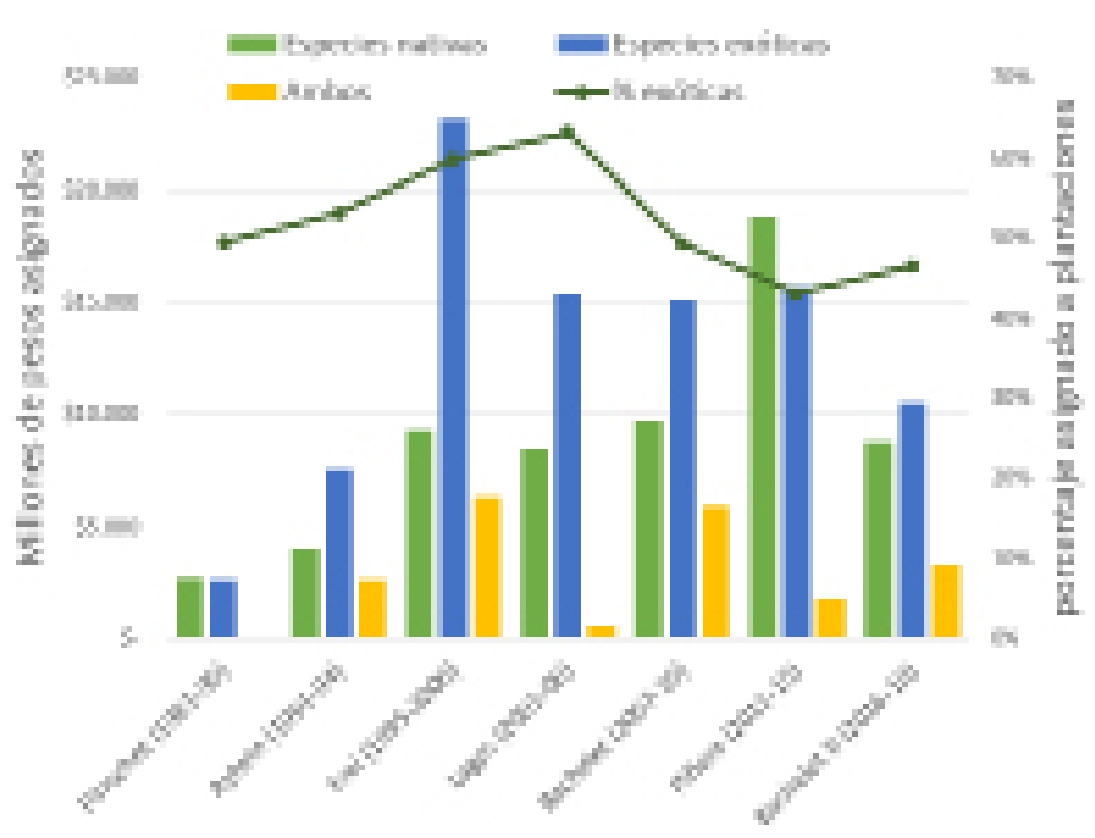

Figura 4. Serie temporal de la inversión estatal en millones de pesos ajustados por inflación en proyectos de investigación y desarrollo en especies nativas, exóticas y ambas desde 1981 hasta 2018. Montos ajustados por inflación. Fuente: Elaboración propia.

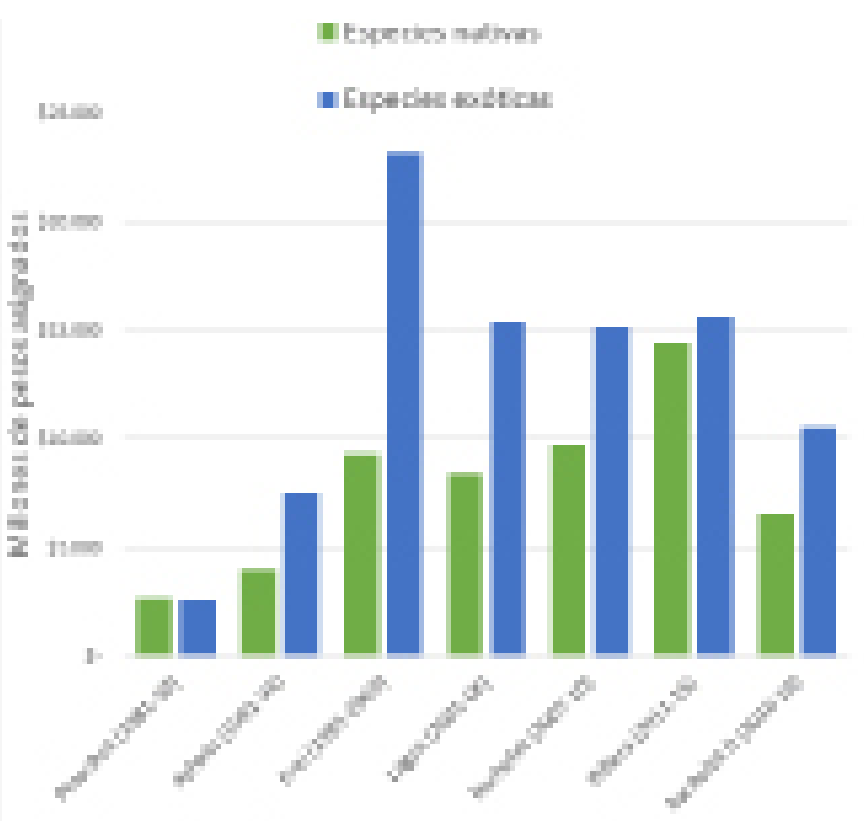

Figura 5. Serie temporal de la inversión estatal en proyectos de investigación y desarrollo en especies nativas, exóticas y ambas desde 1981 hasta 2018 descontando el FIBN. Montos ajustados por inflación. Fuente: Elaboración propia. 


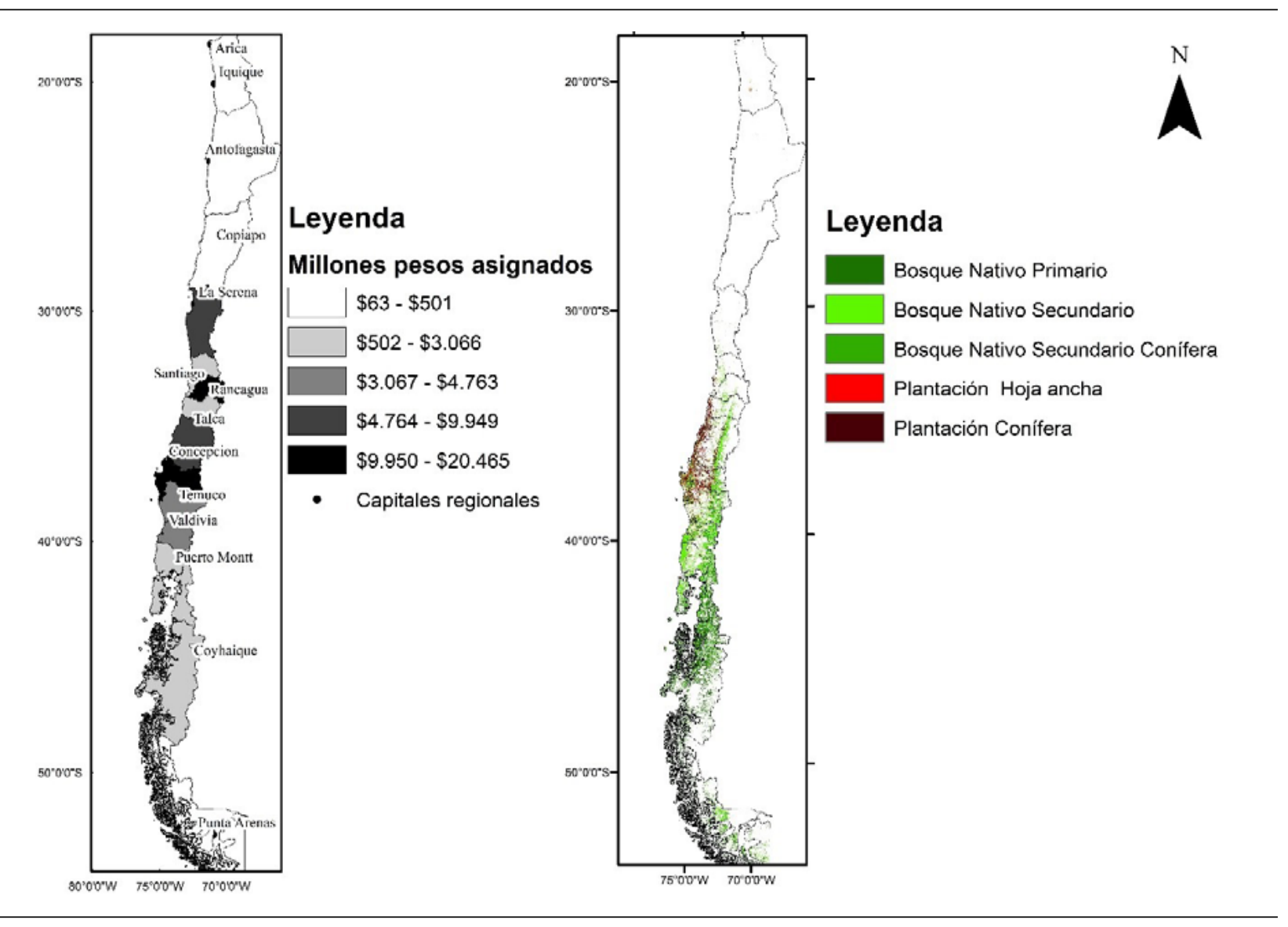

Figura 6. Gasto en millones de pesos asignados desde 1981 hasta 2018 y distribución espacial de las coberturas de bosques y plantaciones. Fuente: Elaboración propia en base a Zhao (2016).

de pesos chilenos Fig. 4). Al analizar temporalmente los proyectos por gobierno, podemos ver que durante el período presidencial de Eduardo Frei Ruiz-Tagle fue cuando más se invirtió en especies exóticas, con un monto total de US\$32,408,392 (\$23,172 millones de pesos Fig. 4). El mayor porcentaje de inversión estatal centrado en especies exóticas fue durante el mandato de Ricardo Lagos que llegó a ser el 62\% de las inversiones. Desde el primer gobierno de Michelle Bachelet, decae la proporción de proyectos asignado a plantaciones forestales. Luego, desde el primer gobierno de Sebastián Piñera, en el año 2010, el monto total dedicado al sector bosques nativos es mayor que el de plantaciones, tendencia que se revierte con el segundo gobierno de Michelle Bachelet (Fig. 4).

El aumento de fondos para el bosque nativo se relaciona con la entrada en vigor del Fondo de Investigación del Bosque Nativo (FIBN) de la ley de Bosque Nativo 20.283, que se ejecutó por primera vez en 2010. De hecho, al descontar el monto asignado por este instrumento, el aparente incremento en la inversión en investigación y desarrollo en bosques nativos desde 2010 no es tal y, en todos los períodos presidenciales, la inversión en plantaciones forestales es mayor a los bosques nativos (Fig. 5).

\section{Asignación regional}

Al comparar la distribución de los bosques con las asignaciones de dinero podemos ver que estas están concentradas en la región de BioBío (capital Concepción) y Metropolitana, mientras los bosques están entre la región de la Araucanía y los Lagos, es decir por debajo de $\operatorname{los} 40^{\circ}$ S (Fig. 6).

En cuanto a la distribución de recursos totales asignados por región, estos están concentrados en la región Metropolitana, con capital en Santiago, y la región del BioBío (Fig. 7). Para poder visualizar en una sola escala numérica los gastos en especies nativas y exóticas fue necesario llevar todos los montos a una escala logarítmica. Al analizar el sector de especies nativas, los proyectos se centraron entre las regiones del Maule y la Araucanía. En cuanto a las especies 

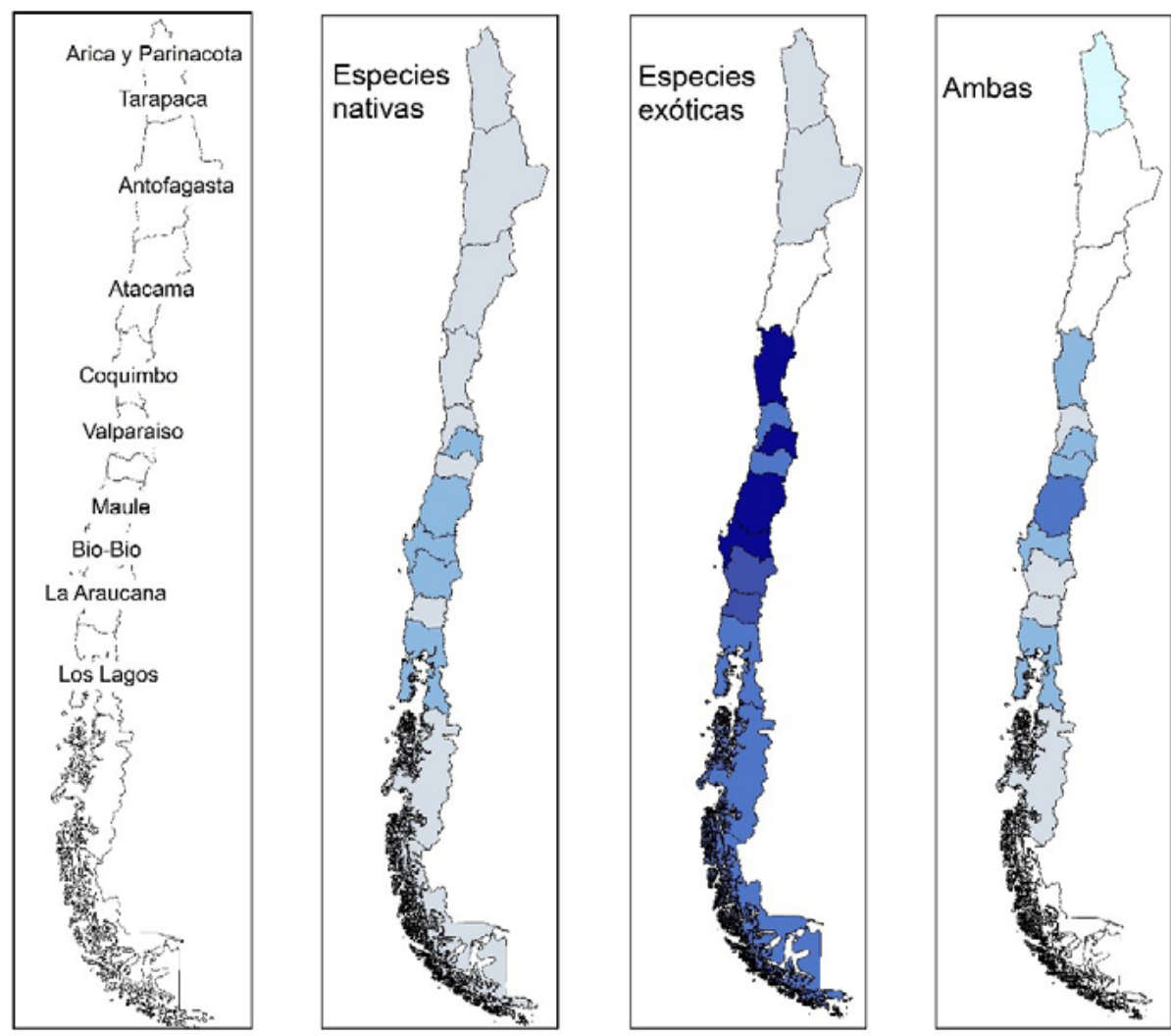

\section{Leyenda}



Figura 7. Asignación a cada región de montos totales, por especies asignados desde 1981 hasta 2018. Montos ajustados por inflación. Nótese que los montos en azul están en escala logarítmica para poder hacerlos comparables. Fuente: Elaboración propia.

exóticas, estos se centraron entre la región del Maule y el BioBío (Fig. 6 y 7). La presencia de proyectos con especies exóticas en la zona centro norte corresponden a desarrollo tecnológico de introducción de especies como el eucalipto, aromo y argán, ejecutados por el Instituto Forestal en la región de Coquimbo, además de proyectos relacionados con uso de maquinaria y mejoramiento genético. En contraste, los proyectos que se desarrollaron relacionados con bosques nativos en la zona norte del país están relacionados con ensayos experimentales de algarrobo y palma para sectores rurales. En la región del Maule es donde se ejecutaron más proyectos de especies nativas como exóticas. Solo un proyecto da cuenta de un $32 \%$ de este monto, interesantemente, y fue un FONDEF de prevención de incendios ejecutado por la Universidad de Chile adjudicado en 1992. Todos los otros proyectos tienen relación con mejoramiento de la gestión sustentable y certificación de manejo de bosques y plantaciones forestales.

\section{Discusión}

El objetivo de este trabajo fue saber cuánto ha invertido el Estado en investigación y desarrollo en especies nativas, principalmente forestales, y en especies exóticas, principalmente especies de rápido crecimiento. El objetivo fue comprender cómo, a lo largo de siete gobiernos y por treinta y siete años, el Estado ha asignado recursos a dos sectores que serán críticos en la mitigación del cambio climático. Se encontró que la inversión estatal ha privilegiado la generación de conocimiento y desarrollo basado en especies exóticas, con porcentajes que superan la mitad de los fondos asignados.

En un estudio anterior, Donoso \& Otero (2005) analizaron la inversión fiscal a través de proyectos FONDEF y FONDECYT, entre 2001 y 2004. Los autores encontraron que se invirtió 3,5 veces más en plantaciones forestales que en bosques nativos. En el presente trabajo, se analizaron 1.139 proyectos entre 1981 y 2018 y se encontró que esta 
razón es de 1,5 veces. Sin embargo, si es que se remueve el FIBN, esta razón sería de 1,7 veces. Ante esto parece ser que este fondo juega un rol fundamental en cuanto a nivelar levemente la inversión pública en ambos sectores, pero el poco tiempo que ha estado en funcionamiento difícilmente impacta en las posibles decisiones que tome el pequeño y mediano propietario hoy.

Ya está largamente documentado cómo las políticas de Estado en el pasado, tales como créditos, transferencias de capitales, políticas macroeconómicas y subsidios han permitido construir un sector basado en plantaciones que ha sido tan rentable (Camus, 2006; Clapp, 1995; Cubbage et al., 2014; Manuschevich, 2018; Niklitschek, 2007). La ventaja con la que hoy corren las plantaciones exóticas de rápido crecimiento, se creó mediante una serie de políticas públicas (Clapp, 1995). Es importante recordar que el sector forestal basado en especies de rápido crecimiento no solo se cimentó en base al subsidio forestal, sino que también fueron fundamentales los cambios macroeconómicos que sufrió el país desde 1976 (Niklitschek, 2007). La intención de este trabajo es aportar a la comprensión de cómo, desde la década de 1980 en adelante, se han orientado los esfuerzos y recursos fiscales por medio de la investigación y el desarrollo para mantener a este sector tan rentable, que desafió la idea de que las plantaciones forestales son naturalmente las más competitivas del mundo. La alta rentabilidad de las plantaciones forestales existe, justamente, gracias a las relaciones de comercio que se establecieron en la década de 1990, producto de la firma de tratados de libre comercio por parte de los gobiernos de la Concertación (Niklitschek, 2007).

En este sentido resulta especialmente interesante que el período en el que más se asignaron fondos a las especies exóticas fue durante el segundo gobierno de la Concertación (1994-2000). El gobierno de Frei Ruiz-Tagle fue también el fin de un período de consolidación de una economía exportadora de materias primas (Moguillansky, 1999). El gasto en investigación y desarrollo en especies exóticas resulta coherente con un proyecto político mayor: un proyecto de una economía exportadora de materias primas (Liverman \& Vilas, 2006). Al mismo tiempo que se buscaban nuevos mercados para vender materias primas, se invertía en hacer que esta producción fuera mayor, mediante la inversión en tecnología. De esta forma, confirmamos lo que propone Peck (2001): el Estado neoliberal no está ausente, está muy presente, especialmente en las coyunturas clave del proyecto que construye. Por otro lado, es notoria la concentración espacial de los fondos asignados para especies exóticas: los mayores montos se concentran en las regiones del
Maule, Ñuble y BioBío, además de Santiago. De esta forma se han construido regiones dedicadas a la producción de solo uno o dos comodities. Las regiones de Maule, Ñuble y BioBío son conocidas por estar dominadas por plantaciones forestales. Nuevamente, la especialización de la producción no es natural, sino que producida por políticas de Estado. Entre ellas la asignación de recursos fiscales para investigación y desarrollo de forma que estas regiones sean las más competitivas.

La discusión actual sobre las medidas de mitigación más adecuadas para enfrentar el cambio climático ha planteado que la mejor opción es la forestación con especies nativas y la recuperación de bosques degradados, más que las plantaciones forestales de rápido crecimiento. Los bosques entregan múltiples beneficios además de secuestro de carbono, por lo que debieran ser el foco de los esfuerzos privados y públicos (Chazdon \& Brancalion, 2019). La evidencia científica en Chile muestra que los bosques -si es que se les permite crecer- ayudan a regular los caudales, controlar la erosión y mantienen la biodiversidad (Alvarez-Garreton, Lara, Boisier \& Galleguillos, 2019; Banfield, Braun, Barra, Castillo \& Vogt, 2018; Braun et al., 2017; Iroumé, Mayen \& Huber, 2006; Little, Lara, McPhee \& Urrutia, 2009; C. Oyarzún, Campos \& Huber, 1997; Oyarzún \& Huber, 2003). Según Alvarez-Garretón et al., (2019) si se forestaran solo 100 hectáreas con especies exóticas, podríamos observar una disminución en la disponibilidad hídrica del $45 \%$ en la zona centrosur del país, lo que puede exacerbarse aún más en un contexto de disminución de las precipitaciones por efecto del cambio climático. Como se está planteando la nueva ley de fomento forestal, que propone un nuevo subsidio independiente de la especie en un contexto tecnológico y productivo donde por más de treinta y siete años se ha privilegiado la investigación y el desarrollo de especies exóticas, difícilmente podría producir un paisaje más resiliente al cambio climático.

Por otra parte, las políticas de mitigación no pueden obviar los posibles impactos sociales. Como se mencionó, la forestación masiva puede exacerbar la migración a la cuidad y el despoblamiento del campo. En general, los pequeños propietarios producen leña para venta y autoconsumo (Burschel, Hernández \& Lobos, 2003). A pesar de que esta se tiende a asociar con algo poco masivo, a nivel mundial, esta fuente de energía representa el 10\% de la producción de energía primaria mundial (IEA, 2016). Además, es una fuente de energía producida por campesinos y comprada por personas de ingresos bajos o medios, ya que, por unidad de calor, es la más barata. Adicionalmente, la producción de leña no implica el 
consumo de combustibles fósiles para calefaccionarse (Burschel et al., 2003). En este sentido, es fundamental que la investigación y desarrollo se oriente a este sector, que tiene impactos en la deforestación y degradación del bosque, por el mal manejo, y en la calidad del aire por la combustión con escasa tecnología en pueblos y ciudades. Aquí la investigación y desarrollo con orientación pública pueden ser de gran aporte.

\section{Limitaciones del estudio y futuros estudios}

Este análisis se basó en gran medida en los datos secundarios entregados por la base de datos OPIA. Si bien estos fueron verificados en la mayoría de los casos, no se pueden desestimar errores. La variable de región de ejecución se codificó en base a la ubicación de la sede del organismo ejecutor, por ejemplo, un proyecto FONDECYT sobre bosque nativo adjudicado por la Universidad de Chile, aparece localizado en la región Metropolitana, aunque parte de la investigación se puede haber realizado en alguna otra parte. Por otro lado, solo se consideran los montos que se asignaron, lo cual no necesariamente es una medida de la calidad e impacto del proyecto o fondo, pero es una aproximación cuantificable.

En el futuro sería interesante comprender el propósito de los proyectos, además de la especie, de manera tal de comprender cuánto se ha gastado, por ejemplo, en aumentar la productividad en especies exóticas y nativas, y contrastarlo con el gasto en asociatividad campesina o desarrollo de tecnologías no contaminantes. Estos datos permitirían comprender de una manera más fina los lineamientos que ha desarrollado el Estado en estos últimos treinta y siete años.

\section{Conclusión}

Analizar en la inversión estatal en investigación y desarrollo de especies nativas y exóticas es fundamental para comprender el contexto tecnológico y productivo en el cual se desenvolverían las propuestas de mitigación basadas en el sector forestal de cara a la COP25. En el caso de Chile, el desarrollo tecnológico y productivo durante los últimos treinta y siete años ha estado basado en privilegiar a las especies exóticas por sobre las especies nativas, pues se ha invertido 1,5 veces más en las primeras. Si se considera la alta rentabilidad privada de las plantaciones exóticas y que el Estado ha favorecido la producción de conocimiento y tecnología sobre especies exóticas, difícilmente se podría argumentar que las especies que se plantarían con un nuevo subsidio son el resultado de la libre elección de los pequeños y medianos propietarios.
En este contexto tecnológico y productivo, una nueva ley de fomento forestal basada únicamente en un nuevo subsidio independiente de la especie probablemente impulsará nuevamente la expansión de las plantaciones exóticas, lo que exacerba la vulnerabilidad socioambiental de los territorios forestales. La Conferencia de las Partes (COP-25) es una instancia política, independiente de cuándo y dónde se realice, tendrá repercusiones en el largo plazo para el desarrollo del país y el bienestar de las generaciones futuras $\mathrm{y}$, en un contexto de cambio climático, se requiere reconsiderar aquellas políticas a la luz de los beneficios sociales que pueden entregar en el largo plazo.

\section{Referencias bibliográficas}

Alvarez-Garreton, C., Lara, A., Boisier, J. P. \& Galleguillos, M. (2019). The Impacts of Native Forests and Forest Plantations on Water Supply in Chile. Forests, 10(6), 473. https://doi.org/10.3390/ f10060473

Andersson, K., Lawrence, D., Zavaleta, J. \& Guariguata, M. R. (2016). More trees, more poverty? The socioeconomic effects of tree plantations in Chile, 2001-2011. Environmental management, 57(1), 123136. https://doi.org/10.1007/s00267-015-0594-X

Arrow, K. (1962). Economic Welfare and the Allocation of Resources for Invention (pp. 609-626) [NBER Chapters]. Recuperado de https://econpapers.repec. org/bookchap/nbrnberch/2144.htm

Banfield, C. C., Braun, A. C., Barra, R., Castillo, A. \& Vogt, J. (2018). Erosion proxies in an exotic tree plantation question the appropriate land use in Central Chile. CATENA, 161, 77-84. https://doi. org/10.1016/j.catena.2017.10.017

Bernstein, H. (2002). Agrarian classes in capitalist development. En Capitalism and development (pp. 54-85). Routledge.

BMEL. (2019). The BMEL's research institutions. Recuperado de https://www.bmel.de/EN/Ministry/ Research-Innovation/_Texte/Research-InstitutesBMELV.html

Borras, S. M. \& Franco, J. C. (2018). The challenge of locating land-based climate change mitigation and adaptation politics within a social justice perspective: Towards an idea of agrarian climate justice. Third 
World Quarterly, 39(7), 1308-1325. https://doi.org/1 $0.1080 / 01436597.2018 .1460592$

Braun, A. Ch., Troeger, D., Garcia, R., Aguayo, M., Barra, R. \& Vogt, J. (2017). Assessing the impact of plantation forestry on plant biodiversity: A comparison of sites in Central Chile and Chilean Patagonia. Global Ecology and Conservation, 10, 159-172. https://doi.org/10.1016/j.gecco.2017.03.006

Burschel, H., Hernández, A. \& Lobos, M. (2003). Leña: Una fuente energética renovable para Chile. Santiago de Chile: Editorial Universitaria.

Camus, P. (2006). Ambiente, Bosques y Gestion Forestal en Chile: 1541-2005. Santiago de Chile: LOM Ediciones.

Cárdenas,A. \& Antileo, E. (2006). Plantaciones forestales en Chile y su relación con el pueblo Mapuche. Foro Social de Resistencia contra los Agronegocios. Presentado en Buenos Aires, Argentina. Buenos Aires, Argentina.

Carmona, M. R., Aravena, J. C., Bustamante-Sanchez, M. A., Celis-Diez, J. L., Charrier, A., Diaz, I. A et. al. (2010). Senda Darwin Biological Station: Long-term ecological research at the interface between science and society. Revista Chilena de Historia Natural, 83(1), 113-142.

Chazdon, R. \& Brancalion, P. (2019). Restoring forests as a means to many ends. Science, 365(6448), 24-25. https://doi.org/10.1126/science.aax9539

Clapp. (1998). Regions of refuge and the agrarian question: Peasant agriculture and plantation forestry in Chilean Araucania. World Development, 26, 571-589.

Clapp, R. A. (1995). Creating Competitive Advantage: Forest Policy as Industrial Policy in Chile. Economic Geography, 71, 273-296.

Cubbage, F., Mac Donagh, P., Balmelli, G., Morales Olmos, V., Bussoni, A., Rubilar, R. \& Carrero, O. (2014). Global timber investments and trends, 20052011. New Zealand Journal of Forestry Science, 44(1), S7. https://doi.org/10.1186/1179-5395-44S1-S7
Cubbage, F., Mac Donagh, P., Sawinski Junior, J., Rubilar, R., Donoso, P., Ferreira, A.\& Alvarez, J. (2007). Timber investment returns for selected plantations and native forests in South America and the Southern United States. New Forests, 33, 237255. https://doi.org/10.1007/s11056-006-9025-4

Donoso, P. \& Otero, L. (2005). Hacia una definición de país forestal: ¿Donde se sitúa Chile? Bosque, 26, 5-18.

Echeverria, C., Coomes, D., Salas, J., Rey-Benayas, J. M., Lara, A. \& Newton, A. (2006). Rapid deforestation and fragmentation of Chilean Temperate Forests. Biological Conservation, 130(4), 481-494. https:// doi.org/10.1016/j.biocon.2006.01.017

FAO. (2004). Trends and current status of the contribution of the forestry sector to national economies $\left(\mathrm{N}^{\circ}\right.$ Working paper: FSFM/ACC/07). Recuperado de http://www.fao.org/3/ad493e/ad493e05.htm

FAO. (2019). FAO statistics. Recuperado de http://www. fao.org/corp/statistics/en/

Fernow, B. E. \& Bernhard E. (1911). A brief history of forestry: In Europe, the United States and other countries. Recuperado de http://archive.org/details/ briefhistoryoffo00fern

FIA. (2019). Banco de Proyectos. Recuperado https:// www.opia.cl/601/w3-propertyvalue-71885.html

Franco, J. C. \& Borras, S. M. (2019). Grey areas in green grabbing: Subtle and indirect interconnections between climate change politics and land grabs and their implications for research. Land Use Policy, 84, 192-199. https://doi.org/10.1016/j. landusepol.2019.03.013

Fuglie, K., Ballenger, N., Rubenstein, K. D., Klotz, C., Ollinger, M., Vasavada, U. \& Yee, J. (1996). Agricultural Research and Development: Public and Private Investments Under Alternative Markets and Institutions. Recuperado de http://www.ers.usda.gov/ publications/pub-details/?pubid $=40696$

Gerber, J. F. (2011). Conflicts over industrial tree plantations in the South: Who, how and why? Global Environmental Change, 21(1), 165-176. https://doi. org/10.1016/j.gloenvcha.2010.09.005 
Gobierno de Chile. (2015). Contribución nacional tentativa de Chile (INDC) para el acuerdo climático Paris 2015. Recuperado de https://www4.unfccc.int/ sites/submissions/INDC/Published\%20Documents/ Chile/1/Chile\%20INDC\%20FINAL.pdf

Goverment of Canada. (2019). About the Canadian Forest Service. Recuperadode https://www.nrcan. gc.ca/our-natural-resources/forests-forestry/aboutcanadian-forest-service/17545

Guerra Martínez, A. (2019). US\$ 37,5 millones al año para fomento forestal. Revista Campo.

Hartmann, D., Guevara, M. R., Jara-Figueroa, C., Aristarán, M. \& Hidalgo, C. A. (2017). Linking Economic Complexity, Institutions, and Income Inequality. World Development, 93, 75-93. https:// doi.org/10.1016/j.worlddev.2016.12.020

Heilmayr, R., Echeverría, C., Fuentes, R. \& Lambin, E. F. (2016). A plantation-dominated forest transition in Chile. Applied Geography, 75, 71-82. https://doi. org/10.1016/j.apgeog.2016.07.014

Hidalgo, C. A. \& Hausmann, R. (2009). The building blocks of economic complexity. Proceedings of the National Academy of Sciences, 106(26), 1057010575. https://doi.org/10.1073/pnas.0900943106

IEA. (2016). World Energy Outlook 2016 [Text]. Recuperado de https://www.oecd-ilibrary.org/energy/ world-energy-outlook-2016_weo-2016-en

Iroumé, A., Mayen, O. \& Huber, A. (2006). Runoff and peak flow responses to timber harvest and forest age in southern Chile. Hydrological Processes, 20(1), 37-50. https://doi.org/10.1002/hyp.5897

Kile, G. A., Nambiar, E. K. S. \& Brown, A. G. (2014). The rise and fall of research and development for the forest industry in Australia. Australian Forestry, 77(3-4), 142-152. https://doi.org/10.1080/00049158. 2014.990867

Little, C., Lara, A., McPhee, J. \& Urrutia, R. (2009). Revealing the impact of forest exotic plantations on water yield in large scale watersheds in South-Central Chile. Journal of Hydrology, 374, 162-170. https:// doi.org/10.1016/j.jhydrol.2009.06.011
Liverman, D. M. \& Vilas, S. (2006). Neoliberalism and the environment in Latin America. Annu. Rev. Environ. Resour, 327-63.

Mansfield, B., Munroe, D. K. \& McSweeney, K. (2010). Does Economic Growth Cause Environmental Recovery? Geographical Explanations of Forest Regrowth. Geography Compass, 4(5), 416-427. https://doi.org/10.1111/j.1749-8198.2010.00320.x

Manuschevich, D. (2018). A Critical Assessment of the Adaptive Capacity of Land Use Change in Chile: A Socio-Ecological Approach. Land Use - Assessing the Past, Envisioning the Future. https://doi.org/10.5772/ intechopen.80559

Mather, A. S. \& Needle, C. (1998). The forest transition: A theoretical basis. Area, 30 (2), 117-124. https://doi. org/10.1111/j.1475-4762.1998.tb00055.x

McWethy, D. B., Pauchard, A., García, R. A., Holz, A., González, M. E., Veblen, T. T. \& Currey, B. (2018). Landscape drivers of recent fire activity (2001-2017) in south-central Chile. PLOS ONE, 13(8), e0201195. https://doi.org/10.1371/journal.pone.0201195

Miranda, A., Altamirano, A., Cayuela, L., Lara, A. \& González, M. (2017). Native forest loss in the Chilean biodiversity hotspot: Revealing the evidence. Regional Environmental Change, 17(1), 285-297. https://doi.org/10.1007/s10113-016-1010-7

Miranda, A., Lara, A., Altamirano, A., ZamoranoElgueta, C., Hernández, J., González, M. \& Promis, A. (2018). Monitoreo de la superficie de los bosques nativos de Chile: Un desafío pendiente. Bosque (Valdivia), 39(2), 265-275. https://doi.org/10.4067/ s0717-92002018000200265

Moguillansky, G. (1999). La inversión en Chile: ¿el fin de un ciclo de expansión? Recuperado de https:// repositorio.cepal.org//handle/11362/2084

Munroe, D. K., McSweeney, K., Olson, J. L. \& Mansfield, B. (2014). Using economic geography to reinvigorate land-change science. Geoforum, 52, 1221. https://doi.org/10.1016/j.geoforum.2013.12.005

Niklitschek, M. E. (2007). Trade liberalization and land use changes: Explaining the expansion of afforested land in Chile. Forest Science, 53, 385-394. 
Oyarzún, C., Campos, H. \& Huber, A. (1997). Exportación de nutrientes en microcuencas con distinto uso del suelo en el sur de Chile (Lago Rupanco, X Región). Revista Chilena de Historia Natural, 70, 507-519.

Oyarzún, C. E. \& Huber, A. (2003). Nitrogen export from forested and agricultural watersheds of southern Chile. Gayana Bot, 60(1), 63-68.

Peck, J. (2001). Neoliberalizing states: Thin policies/hard outcomes. Progress in Human Geography, 25(3), 445455. https://doi.org/10.1191/030913201680191772

Simoes, A. J. G. \& Hidalgo, C. A. (2011). The Economic Complexity Observatory: An Analytical Tool for Understanding the Dynamics of Economic Development. Scalable Integration of Analytics and Visualization. Recuperado de https://www.researchgate.net/profile/Cesar Hidalgo/publication/221605462 The Economic Complexity_Observatory_An_Ānalytical_Tool_ for_Understanding the_Dynamics_of_Economic Development/links/54f472430cf24eb8794e8a6d.pdf

Thünen Institute. (2019). Johann Heinrich von Thünen Institute Federal Research Institute for Rural Areas, Forestry and Fisheries. Recuperado de https://www. thuenen.de/en/about-us/the-institute/

USFS. (2019). About R\&D - US Forest Service Research \& Development. Recuperado de https://www.fs.fed. us/research/about/

World Bank. (2018). DataBank. Recuperado de https:// datos.bancomundial.org/

Zhao, Y., Feng, D., Yu, L., Wang, X., Chen, Y., Bai, Y.\& Gong, P. (2016). Detailed dynamic land cover mapping of Chile: Accuracy improvement by integrating multi-temporal data. Remote Sensing of Environment, 183, 170-185. https://doi.org/10.1016/j. rse.2016.05.016

\section{Apéndice}

Esquema de clasificación para todas las bases de datos $0=$ sin interés para este trabajo, como, por ejemplo: desarrollo de maceteros biodegradables, desarrollo de frutales, proyectos centrados en bioremediación, pastizales, giras técnicas, asistencias a congresos. Se excluyeron los proyectos que estudien suelos a menos que estén en relación con las distintas coberturas vegetales. También se excluyen premios.

1 = bosques nativos, vegetación nativa, incluyendo hongos, microorganismos, flores y berries nativos (e.g.: Ugni molinae)

2 = especies exóticas, incluyendo pino, eucalipto, atriplex, paulownia u otras especies exóticas, excluyendo frutales.

3 = implican especies nativas y exóticas, tales como proyectos de combate de incendios, uso de suelo o planificación espacial y productos forestales no madereros a menos que se explicite que se trata únicamente de especies nativas. 